\title{
Comparative proteomic analysis of secreted proteins from nasopharyngeal carcinoma-associated stromal fibroblasts and normal fibroblasts
}

\author{
SHENGLEI GE ${ }^{1}$, YITAO MAO ${ }^{1}$, YAN $^{2}{ }^{2}$, DINGHUA XIE ${ }^{1},{\text { ZHUCHU } \text { CHEN }^{3} \text { and ZHIQIANG XIAO }}^{3}$ \\ Departments of ${ }^{1}$ Otolaryngology - Head and Neck Surgery, and ${ }^{2}$ Hematology, Second Xiang-ya Hospital, \\ Central South University, Changsha; ${ }^{3}$ Institute of Oncology, Xiang-ya Medical College, \\ Central South University, Changsha, Hunan, P.R. China
}

Received December 13, 2011; Accepted January 30, 2012

DOI: $10.3892 /$ etm.2012.483

\begin{abstract}
Since the concept of the secretome (ensemble of proteins secreted and/or shed from cells) was proposed, it has become an attractive and challenging proteomic technology in recent years. However, secretome analysis still faces some difficulties mainly related to sample collection and preparation. In the present study, we established a reliable method for extracting secreted proteins by ultrafiltration centrifugation and conducting secretomic analysis. Accumulating evidence suggests that carcinoma-associated stromal fibroblasts (CAFs) play an important role in cancer initiation and progression. To investigate the expression patterns of secreted proteins from fibroblasts and to identify the secreted proteins involved in nasopharyngeal carcinoma (NPC) carcinogenesis, we conducted comparative secretome analysis between CAFs and normal fibroblasts. After two-dimensional gel electrophoresis (2-D PAGE), 11 significant spots were differentially expressed and identified by matrix-assisted laser desorption/ionization time-of-flight (MALDI-TOF) mass spectrometry (MS). These proteins may take part in the regulation of the tumor microenvironment through different processes. The expression level of galectin-1 in the CAF supernatant was also determined by ELISA. This study provides useful information and new clues for the further understanding of the regulatory mechanisms of CAFs in the NPC microenvironment.
\end{abstract}

\section{Introduction}

As the most abundant cells in tumor stroma, tumor-associated fibroblasts or carcinoma-associated stromal fibroblasts (CAFs) have distinctly different morphological and biological charac-

Correspondence to: Dr Yan Yi, Department of Hematology, Second Xiang-ya Hospital, Central South University, 139 Middle Renmin Road, Changsha, Hunan 410011, P.R. China

E-mail: yiyan_yi@yahoo.com.cn

Key words: nasopharyngeal carcinoma, fibroblast, secreted protein, secretome teristics compared with normal fibroblasts (NFs). CAFs may promote the malignant transformation of epithelial cells by secreting a variety of growth factors, cytokines, chemoattractants and enzymes. Scatter factor/hepatocyte growth factor, insulin growth factor, matrix metalloproteinases and fibroblast activation protein have been confirmed to play an important role in tumor-host crosstalk (1-3). Accumulating evidence suggests that CAFs play an important role in cancer initiation and progression (4). Co-culture system or three-dimensional (3D) culture system has been used to study the interaction between CAFs and cancer cells in recent years. However, the study of cells in 3D cultures has been hampered by the lack of simple methods to analyze them. Current techniques are timeconsuming, require expensive equipment such as confocal microscopy for optimal results, and are poorly adapted for study $(5,6)$. Moreover, these tumor-promoting properties of CAFs appear to be partially independent of the presence of tumor cells and are maintained in vitro even in the absence of epithelial cells $(1,7)$. Therefore, it is feasible to isolate fibroblasts from carcinoma specimen and to culture in vitro for understanding the role of CAFs in the tumor microenvironment. Since the concept of the secretome (ensemble of proteins secreted and/or shed from cells) was proposed (8), it has become an attractive and challenging proteomic technology in recent years. However, secretome analysis still faces some difficulties mainly related to sample collection and preparation. In the present study, we explored one method of sample preparation, and we conducted secretome analysis of CAFs and NFs to evaluate the expression patterns of secreted proteins from fibroblasts in NPC carcinogenesis. The experimental results will provide a basis for the further understanding of the regulatory mechanisms of CAFs in the NPC microenvironment.

\section{Materials and methods}

Nasopharyngeal mucosal samples. All experimental procedures in this study were approved by the Ethics Committee of the Central South University School of Medicine. In total, 8 nasopharyngeal mucosal samples from patients with poorly differentiated nasopharyngeal squamous cell carcinoma and 
8 from patients with sinusitis were obtained with informed consent at the Second Xiangya Hospital of Central South University. Diagnoses were pathologically confirmed, and none of the patients had received prior chemotherapy or radiation therapy.

Fibroblast culture. CAFs and NFs were obtained by tissue culture as described in detail elsewhere (9). Nasopharyngeal mucosa tissue was washed extensively with sterile PBS to remove contaminating debris and red blood cells, and cut into small pieces. Then cells were dissociated by $0.25 \%$ trypsin (Sigma, St. Louis, MO, USA) and purified using a curettage method combined with trypsinization. The outgrowing fibroblasts were cultivated with RPMI-1640 medium (Gibco, Life Technologies Inc, Grand Island, NY, USA) supplemented with $10 \%$ fetal bovine serum (FBS) and $1 \%$ penicillin/streptomycin (Sigma) in an incubator set at $37^{\circ} \mathrm{C}$ and $5 \% \mathrm{CO}_{2}$. Cells were transferred and plated into T-75 flasks. While cells were grown to $70 \%$ confluence, the culture medium was removed and cells were washed more than 3 times with Hank's solution (Sigma), and then serum-free RPMI-1640 medium supplemented with $5 \mu \mathrm{g} / \mathrm{ml}$ transferrin and $5 \mu \mathrm{g} / \mathrm{ml}$ insulin were added to the culture for $72 \mathrm{~h}$. For all experiments, cells used were passaged no more than three times.

Preparation of secreted protein samples. Culture medium was collected and centrifuged for $10 \mathrm{~min}$ at $1,000 \mathrm{x} \mathrm{g}, 10 \mathrm{~min}$ at $3,000 \times \mathrm{g}$ at $4^{\circ} \mathrm{C}$ to remove cell debris or dead cells. The supernatant was harvested and filtered through a membrane filter (nominal pore size $0.22 \mu \mathrm{m}$ ), and then secreted proteins were enriched and concentrated by ultrafiltration centrifugation (Millipore, Bedford, MA, USA). Finally, approximately $250 \mu 1$ of the supernatant containing secreted proteins was obtained every time according to the CentriPlus Centrifugal Filter Device User Guide. Protein lysis buffer $(2 \mathrm{~mol} / \mathrm{l}$ thiourea, $7 \mathrm{~mol} / 1$ urea, $40 \mathrm{mmol} / 1$ Tris, 4\% CHAPS, $65 \mathrm{~mol} / \mathrm{l}$ DTT) was added to the concentrated supernatant. The mixture was centrifuged for $30 \mathrm{~min}$ at $15,000 \mathrm{x}$ g at $4^{\circ} \mathrm{C}$ and the final supernatant (ie. secreted proteins) was obtained. The protein concentration was measured using a 2D Quantitative kit (Amersham Biosciences, Piscataway, NJ, USA).

2-D PAGE. Aliquots containing approximately $600 \mu \mathrm{g}$ of secreted protein extract were diluted in rehydration buffer for separation by isoelectrical focusing followed by SDS-PAGE (4-15\% gradient gels) exactly in the same manner as previously described elsewhere (10). After SDS-PAGE, the gels were then stained with Coomassie blue staining solution overnight followed by destaining with $18 \%$ methanol and $5 \%$ acetic acid, Triplicate gels were made for each group.

Image analysis. 2-D PAGE images were digitized with an Image scanner (Amersham Biosciences, Piscataway, NJ, USA) and image analysis was conducted using the PDQuest software (Bio-Rad, V7.1) as previously described in detail elsewhere (10).

Identification of proteins by MALDI-TOF MS. Protein spots showing temporal changes in expression in each group were excised and digested in-gel with sequencing-grade modified trypsin (Promega, Madison, WI, USA). Tryptic peptides were separated and analyzed using MALDI-TOF MS. The differentially expressed proteins were identified by peptide mass fingerprint (PMF), SWISS-PROT and NCBI protein databases searching using Mascot Distiller software exactly in the same manner as previously described (10).

Galectin-1 ELISA assay. CAF and NF culture media were collected, respectively, and applied to ELISA assay of galectin-1 in the supernatant.

\section{Results}

2-D PAGE. A reliable method for extracting secreted protein from conditioned medium was established. We isolated CAFs from cancer specimens and cultivated ex vivo for up to 3 passages. Secreted proteins were enriched from the culture supernatant by ultrafiltration centrifugation, and approximately $250 \mu \mathrm{l}$ of the supernatant containing secreted proteins was finally obtained. The 2-D PAGE reference map of the secreted proteins from the fibroblasts was constructed. The differentially expressed proteins were selected and numbered by PDQuestTM (Fig. 1).

Protein identification. Eleven significant spots were identified through MALDI-TOF MS and SWISS-PROT databases (Table I). These secreted protein were consistently expressed in both the CAF and NF supernatants, while 8 were differentially up-regulated in the CAF supernatant when compared with the NF supernatant. The other proteins were down-regulated including cystatin $\mathrm{C}$, complement component $\mathrm{C}$ 1s precursor and heterogeneous nuclear ribonucleoprotein A1. The concentration of galectin-1 in the supernatant was determined by ELISA. We detected a marked difference between the two groups indicating that CAFs may secrete more galectin-1 than NFs.

\section{Discussion}

As it is well known that secreted proteins play a key role in cell signaling, communication and migration, the investigation of secreted proteins has received increased attention in recent years. Secreted proteins are responsible for the crosstalk among cells and understanding this language can largely increase our knowledge concerning the molecular mechanisms of neoplasia. Significant technological advances in the field of proteomics during the last few years have provided shortcuts for the research of secreted proteins, and the concept of the secretome was correspondingly proposed. The secretome has facilitated the study of cell secreted proteins, and it may be a viable strategy for identifying candidate diagnostic and prognostic markers, potential drug and therapeutic targets $(11,12)$. In the present experiment, we explored a set of methods with reference to other studies (13). At first, fibroblast cells were washed repeatedly more than 3 times to eliminate interference of bovine serum albumin, and then serum-free RPMI-1640 medium supplemented with transferrin and insulin as necessary nourishments was added to continue culture. Secondly, the culture medium was harvested and filtered through a $0.22-\mu \mathrm{m}$ membrane filter. Thirdly, the culture medium was concentrated and desalinated by ultrafiltration centrifugation. 

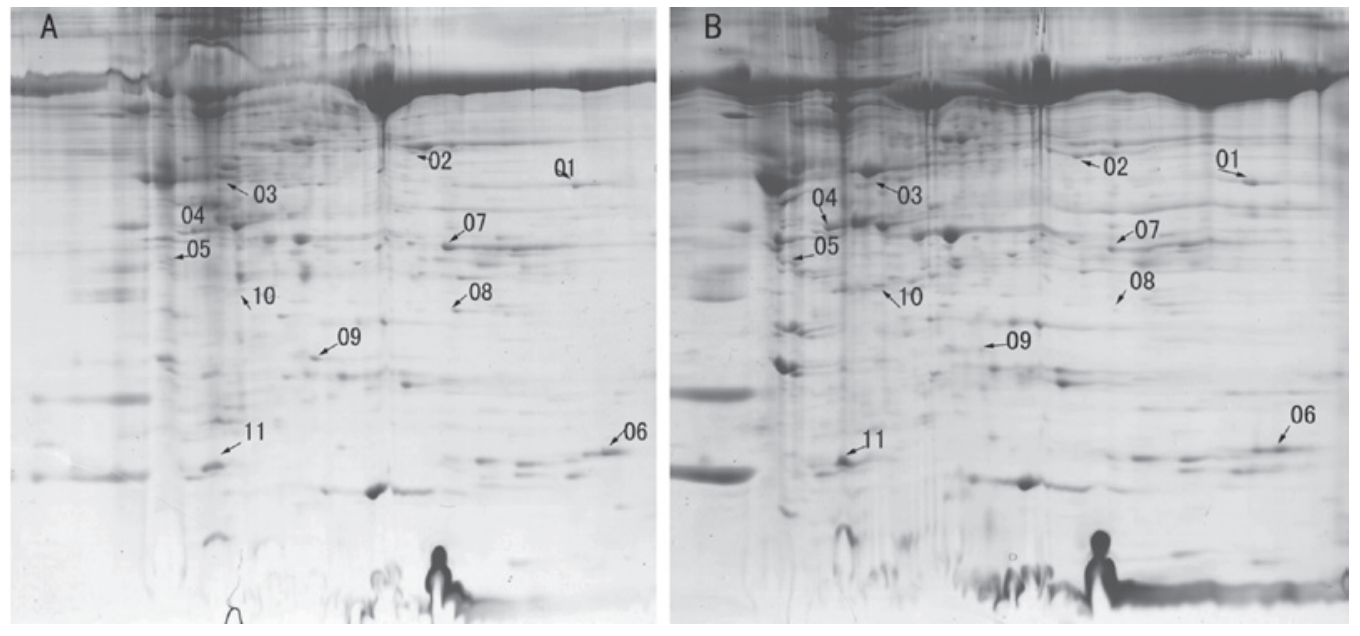

Figure 1. Differentially expressed proteins were selected and numbered. 2-D PAGE of proteins secreted from (A) NFs and (B) CAFs.

Table I. Eleven spots obtained from 2-D PAGE identified via MALDI-TOF MS.

\begin{tabular}{rrlrrr}
\hline No. & Accession no. & \multicolumn{1}{c}{ Protein name } & $\begin{array}{c}\text { Relative molecular } \\
\text { weight (kDa) }\end{array}$ & $\begin{array}{c}\text { Isoelectric } \\
\text { point (pI) }\end{array}$ & $\begin{array}{c}\text { Variable } \\
\text { multiples }^{\mathrm{a}}\end{array}$ \\
\hline 1 & P04075 & Fructose bisphosphate aldolase A & 24.7 & 8.47 & 3.10 \\
2 & P05121 & Plasminogen activator inhibitor 1 & 42.75 & 6.98 & 3.60 \\
3 & P07711 & Cathepsin L & 37.9 & 5.32 & 2.50 \\
4 & P08758 & Membrane annexin A5 & 35.3 & 5.15 & 2.90 \\
5 & P31947 & 14-3-30 protein & 27.7 & 4.69 & 3.20 \\
6 & P01034 & Cystatin C & 12.9 & 7.85 & 0.47 \\
7 & P16035 & Complement component C1s precursor & 78.16 & 4.87 & 0.23 \\
8 & P08294 & Cu/Zn-SOD & 16.2 & 5.85 & 5.20 \\
9 & P09651 & Heterogeneous nuclear ribonucleoprotein A1 & 21.0 & 6.78 & 0.22 \\
10 & P52565 & Rho-GDP dissociation inhibitor 1 & 2.2 & 6.74 & 3.90 \\
11 & P09382 & Galectin 1 & 14.89 & 5.32 & 3.10 \\
\hline
\end{tabular}

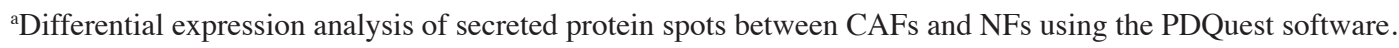

The final step consisted of the lysis and denaturation of the concentrated secreted proteins using a solution composed of $2 \mathrm{~mol} / \mathrm{l}$ thiourea, $7 \mathrm{~mol} / \mathrm{l}$ urea, $4 \% \mathrm{CHAPS}, 40 \mathrm{mmol} / \mathrm{l}$ Tris and $65 \mathrm{~mol} / \mathrm{l}$ DTT.

Although we achieved success in the sample preparation approaches, many problems remain to be solved. The major defect of the method used in this experiment involved the complex operation used to enrich the secreted proteins and the large amount of cell medium required; $50 \mathrm{ml}$ of culture medium was needed for approximately $250 \mu \mathrm{l}$ of the supernatant containing the secreted proteins. In order to enrich the secreted proteins, the supernatant containing the secreted proteins was collected repeatedly and the mixture was repeatedly put through ultrafiltration centrifugation. Obviously, it is extremely important to develop simpler and more efficient approaches such as the nanozeolite-driven approach for enrichment of secretory proteins (14). This technique not only simplifies the operation order, but also saves time. Non-gel electrophoresis technology may also have important applica- tion value in secretome analysis such as nanoproteomics (15), capillary ultrafiltration and multidimensional liquid chromatographic separation in combination with mass spectrometry(16). However, 2-D PAGE which is rapidly being modified plays an irreplaceable role in proteomic analysis and is still the classical and most widely used proteomics method.

Secreted proteins from CAFs may influence the NPC pathological process in different ways. Among these secreted proteins, $\mathrm{Cu} / \mathrm{Zn}-\mathrm{SOD}$ and fructose bisphosphate aldolase $\mathrm{A}$ are associated with cellular oxidative reaction and play an important role in protecting cells against reactive oxygen species injury. Cathepsin L, cystatin C, plasminogen activator inhibitor-1, heterogeneous ribonucleoprotein A1, Rho-GDP dissociation inhibitor-1, 14-3-3 $\sigma$ protein and annexin A5 are associated with signal transduction involved in NPC invasion and metastasis (17-19). As a result, CAFs are involved in the regulation of the NPC microenvironment through these secreted proteins by different processes including protein degradation, cell proliferation, invasion and metastasis. Galectin 1 is one member 
of the family of $\beta$-galactoside-binding proteins implicated in modulating cell-cell and cell-matrix interactions. Galectin 1 may participate in the regulation of the nasopharyngeal carcinoma microenvironment and its regulating mechanism remains unclear and is yet to be further clarified (20).

In conclusion, CAFs may influence the biological behavior of adjacent normal epithelial cells and cancer cells through secreted proteins in an autocrine or paracrine manner to maintain tumor microenvironment balance. Therefore, the fibroblast cell is not a silent character in carcinogenesis, and it plays an important role in this complex process, particularly in the regulation of the tumor microenvironment. Although the $3 \mathrm{D}$ culture system is too complex for operation, its application and investigation warrant future efforts since it may have broad application prospects in the field of secretome analysis of CAFs.

\section{Acknowledgements}

This study was financially supported by the National Natural Science Foundation of China (grant nos. 30700940 and 81100360).

\section{References}

1. Orimo A,Gupta PB,Sgroi DC, Arenzana-Seisdedos F, Delaunay T, Naeem R, Carey VJ, Richardson AL and Weinberg RA: Stroma fibroblasts present in invasive human breast carcinomas promote tumor growth and angiogenesis through elevated SDF-1/CXCL12 secretion. Cell 121: 335-348, 2005.

2. Yang F, Tuxhorn JA, Ressler SJ, McAlhany SJ, Dang TD and Rowley DR: Stromal expression of connective tissue growth factor promotes angiogenesis and prostate cancer tumorigenesis. Cancer Res 65: 8887-8895, 2005.

3. Maeda T, Desouky J and Friedl A: Syndecan-1 expression by stromal fibroblasts promotes breast carcinoma growth in vivo and stimulates tumor angiogenesis. Oncogene 25: 1408-1412, 2006.

4. Bhowmick NA, Neilson EG and Moses HL: Stromal fibroblasts in cancer initiation and progression. Nature 432: 332-337, 2004.

5. Yamada KM and Cukierman E: Modeling tissue morphogenesis and cancer in 3D. Cell 130: 601-610, 2007.

6. Debnath J and Brugge JS: Modelling glandular epithelial cancers in three-dimensional cultures. Nat Rev Cancer 5: 675-688, 2005.

7. Hu M, Yao J, Cai L, Bachman KE, van den Brûle F, Velculescu V and Polyak K: Distinct epigenetic changes in the stromal cells of breast cancers. Nat Genet 37: 899-905, 2005.
8. Tjalsma H, Bolhuis A, Jongbloed JD, Bron S and van Dijl JM: Signal peptide-dependent protein transport in Bacillus subtilis: a genome-based survey of the secretome. Microbiol Mol Biol Rev 64: 515-547, 2000.

9. Sonnenberg M, van der Kuip H, Haubeis S, Fritz P, Schroth W, Friedel G, Simon W, Mürdter TE and Aulitzky WE: Highly variable response to cytotoxic chemotherapy in carcinomaassociated fibroblasts (CAFs) from lung and breast. BMC Cancer 8: 364, 2008.

10. Feng XP, Yi H, Li MY, Li XH, Yi B, Zhang PF, Li C, Peng F, Tang CE, Li JL, Chen ZC and Xiao ZQ: Identification of biomarkers for predicting nasopharyngeal carcinoma response to radiotherapy by proteomics. Cancer Res 70: 3450-3562, 2010.

11. May M: From cells, secrets of the secretome leak out. Nat Med 15: 828, 2009.

12. Pavlou MP and Diamandis EP: The cancer cell secretome: a good source for discovering biomarkers? J Proteomics 73: 1896-1906, 2010.

13. Boraldi F, Bini L, Liberatori S, Armini A, Pallini V, Tiozzo R, Ronchetti IP and Quaglino D: Normal human dermal fibroblasts: proteomic analysis of cell layer and culture medium. Electrophoresis 24: 1292-1310, 2003.

14. Cao J, Hu Y, Shen C, Yao J, Wei L, Yang F, Nie A, Wang H, Shen H, Liu Y, Zhang Y, Tang Y and Yang P: Nanozeolite-driven approach for enrichment of secretory proteins in human hepatocellular carcinoma cells. Proteomics 9: 4881-4888, 2009.

15. Archakov AI, Ivanov YD, Lisitsa AV and Zgoda VG: AFM fishing nanotechnology is the way to reverse the Avogadro number in proteomics. Proteomics 7: 4-9, 2007.

16. Huang CM, Wang CC, Barnes S and Elmets CA: In vivo detection of secreted proteins from wounded skin using capillary ultrafiltration probes and mass spectrometric proteomics. Proteomics 6: 5805-5814, 2006

17. Xu X, Yuan G, Liu W, Zhang Y and Chen W: Expression of cathepsin $\mathrm{L}$ in nasopharyngeal carcinoma and its clinical significance. Exp Oncol 31: 102-105, 2009.

18. Tang CE, Guan YJ, Yi B, Li XH, Liang K, Zou HY, Yi H, Li MY, Zhang PF, Li C, Peng F, Chen ZC, Yao KT and Xiao ZQ: Identification of the amyloid $\beta$-protein precursor and cystatin $\mathrm{C}$ as novel epidermal growth factor receptor regulated secretory proteins in nasopharyngeal carcinoma by proteomics. J Proteome Res 9: 6101-6111, 2010.

19. Yi B, Tan SX, Tang CE, Huang WG, Cheng AL, Li C, Zhang PF, Li MY, Li JL, Yi H, Peng F, Chen ZC and Xiao ZQ: Inactivation of 14-3-3 sigma by promoter methylation correlates with metastasis in nasopharyngeal carcinoma. J Cell Biochem 106: 858-866, 2009

20. Tang CE, Tan T, Li C, Chen ZC, Ruan L, Wang HH, Su T, Zhang PF and Xiao ZQ: Identification of galectin-1 as a novel biomarker in nasopharyngeal carcinoma by proteomic analysis. Oncol Rep 24: 495-500, 2010. 